\title{
Environmental fluctuations drive species' competitive success in experimental invasions
}

\author{
Emmi Räsänen*, Leena Lindström \& Tarmo Ketola
}

\begin{abstract}
Department of Biological and Environmental Science, P.O. Box 35, Fl-40014 University of Jyväskylä, Finland ('corresponding author's e-mail: emmi.j.rasanen@jyu.fi)
\end{abstract}

Received 6 Aug. 2019, final version received 10 Mar. 2020, accepted 13 Mar. 2020

Räsänen, E., Lindström, L. \& Ketola, T. 2020: Environmental fluctuations drive species' competitive success in experimental invasions. - Ann. Zool. Fennici 57: 79-87.

Climate change is presumed to increase both the number and frequency of fluctuations in environmental conditions. Fluctuations can affect the ecological and evolutionary processes that make species more successful competitors. For example, fluctuating conditions can create selection pressures for traits that are profitable in adaptation to fast climate change. On an ecological timescale, environmental fluctuations can facilitate species competitive success by reducing other species' population sizes. Climate change could then enhance species invasions into new areas if fluctuation-adapted invaders displace their native competitors in chancing environments. We tested experimentally whether fast environmental fluctuations, either past (on an evolutionary timescale) or present (on an ecological timescale) affect species competitive success. Bacteria that evolved in either constant or fluctuating temperature were set to compete with the dominant invader Serratia marcescens, which had also evolved in either constant or fluctuating temperature. Moreover, the competition experiments were conducted in environments with similarly constant or fluctuating thermal conditions. The results showed that temperature fluctuations during competition, i.e. on an ecological timescale, made the invader more successful. Surprisingly, we found that the invaders' or its competitor species' evolution in fluctuating environments did not affect the outcome of the competition. Our study highlights the importance of the present environmental fluctuations in promoting species' competitive success and potentially facilitating biological invasions.

\section{Introduction}

It is predicted that as a result of climate change temperatures and also the variability in environmental conditions will increase (IPCC 2018). Global warming has already enhanced the spread of many invasive species (Dukes \& Mooney 1999, Clements \& Ditommaso 2011), but it is also possible that species' evolution under fluc- tuating conditions will contribute to its ability to invade new areas (Lee \& Gelembiuk 2008, Saarinen et al. 2019). This is because fluctuating conditions create selection pressures for traits helping to adapt to fast climate change (Levins 1968, Botero et al. 2015). In addition to increased species' invasiveness, fluctuations in environmental conditions could accelerate global biodiversity loss by making native com- 
munities and their environments more vulnerable to invasions (Parepa et al. 2013, Saarinen et al. 2019). Invasive species are known to be a problem in many ecosystems, and they can, for example, competitively displace native species (Mooney \& Cleland 2001). This calls for studies to predict the success of invasive species under future changing environmental conditions (Ricciardi et al. 2017).

Climatic fluctuations are often associated with increased disturbance in natural environments (Parepa et al. 2013). On an ecological timescale, environmental fluctuations can facilitate species invasions by increasing variation in native species' population sizes, thus reducing competition and releasing resources for invaders to exploit (Davis 2009). Several field and laboratory studies have found disturbed environments to be more prone to invasions than non-disturbed (Burke \& Grime 1996, Davis et al. 2000, Melbourne et al. 2007, Li \& Stevens 2012, Liu et al. 2012). In addition, invasive species have been suggested to originate from areas that are heterogeneous and disposed to disturbances (Baker 1974, Lee \& Gelembiuk 2008, Foucaud et al. 2010, Hufbauer et al. 2012). For example, if the species has evolved in a disturbed environment, it might have pre-adaptations which increase its invasion success in the new environment with similar conditions (Bock 1959, Lee \& Gelembiuk 2008, Hamilton et al. 2015). Human-altered environments are becoming universal, and if species can adapt to this type and intensity of disturbances, they could become successful invaders worldwide. This scenario is known as the anthropogenically induced adaptation to invade hypothesis (AIAI; Hufbauer et al. 2012).

Species evolution under environmental fluctuations, which are fast in relation to species' generation time, might select for characteristics, such as generalism and phenotypic plasticity that make them subsequently successful as invaders (Levins 1968, Meyers et al. 2005, Lee \& Gelembiuk 2008, Ketola et al. 2013, Kristensen et al. 2018). These qualities can increase their ability to tolerate a wide range of conditions; for example, the adaptation to fluctuating temperature by thermal generalism would allow species to prosper in various environments under climate change (Zerebecki \& Sorte 2011). Moreover, the adaptation to fluctuating conditions can make the invader more competitive than the native species (Lee \& Gelembiuk 2008, Duncan et al. 2011). This is true especially if the native species have not adapted to the prevailing fluctuating conditions (Shea \& Chesson 2002). On the other hand, if the native species are also pre-adapted to tolerate fluctuations, the invader might not have a competitive advantage over its local competitors (Saarinen et al. 2019). However, there is little information if the lack of adaptation to fluctuations in native species could make communities less resistant against invasions and increase the risk of extinctions due to competition with the invader (Marvier et al. 2004, Melbourne et al. 2007).

The experimental evolution studies on adaptation to fluctuating environments are numerous (Kassen 2002), yet very few studies exist on the possible effects of fluctuations promoting the biological invasions (Lee \& Gelembiuk 2008). Thus, our aim was to test how rapid temperature fluctuations in the environment during competition (i.e. on an ecological timescale), and during the evolution of both the invader and its competitor species (i.e. on an evolutionary timescale), affect the competitive success of the invader. To investigate the multifactorial nature of invasions, we used several bacterial species that had evolved either in constant or fluctuating temperature and implemented inter-specific competition experiments in similarly constant or fluctuating thermal conditions (Saarinen et al. 2018). With this experimental evolution setup and high levels of replication, we were able to tease apart the effects that are co-occurring in nature.

As an invader we used a strongly competitive species Serratia marcescens, competing with other bacterial species in bicultures. In our competition experiments, the invader started as rare compared with its competitor species. Both the invader and its competitor species were inoculated concurrently, hence having an equal opportunity to use the available resources. Therefore, our experiments reflect asymmetric competition rather than invasion in a strict sense, i.e. when the invader arrives later than its resident species. The level of competitive success of the invader was defined by continuous metrics as the proportion of invader clones in the total number of bacterial clones under specific environmental conditions. 
We hypothesized that: (1) disturbed, thermally fluctuating, environments promote species' competitive success, (2) evolution under fluctuating temperature increases species' competitive success, and (3) species evolved under constant temperature are less resistant to competition in thermally fluctuating environments.

\section{Material and methods}

\section{Study species}

In our study, we used the following four species, all originally obtained from ATCC $^{\circledR}$ (American Type Culture Collection): Serratia marcescens ssp. marcescens $\left(\mathrm{ATCC}^{\circledR} 13880^{\mathrm{TM}}\right)$, Pseudomonas putida (ATCC ${ }^{\circledR} 12633^{\mathrm{TM}}$ ), Pseudomonas fuorescens (ATCC ${ }^{\circledR} 13525^{\mathrm{TM}}$ ) and Novosphingobium capsulatum $\left(\mathrm{ATCC}^{\circledR} 14666^{\mathrm{TM}}\right)$. Bacterial species were chosen based on their abilities to grow well in the same medium and to tolerate rapidly fluctuating temperatures of the experiment. Before the experiments, the clones had evolved for 79 days in thermal cabinets (Lab Companion, ILP-12; Jeio Tech, Seoul, Korea) in two temperature regimes: constant temperature of $30{ }^{\circ} \mathrm{C}$ for the whole period, and fluctuating temperature cycling as follows: $20-30-40-30$ $20{ }^{\circ} \mathrm{C}$ each temperature kept for $2 \mathrm{~h}$ (for details see Saarinen et al. 2018). This corresponded to ca. 86 generations in all species and treatments. The constant temperature was near the optimal temperature for all the bacterial species, when the maximum growth rate and yield were measured (Saarinen et al. 2018). Serratia marcescens was chosen as an invader because it is known to dominate the other study species, i.e. reflecting typical invasive species in this respect, and is also easy to distinguish from the other species when using DNase agar plates (Smith et al. 1969, Ketola et al. 2017).

\section{Competition experiments}

Our study design allowed us to separate the effects of the environment during competition, the evolution of the invader and the evolution of the competitor species (constant vs. fluctuat- ing temperature in all cases) on the competitive success of $S$. marcescens. In this experiment, the competitive success was calculated as the proportion of the $S$. marcescens colonies in the total colony count including also the competitor species colonies. This means that there was no defined threshold value for the competitor species' displacement. The invader clone that had evolved in either constant or fluctuating temperature competed with the competitor species' clone that had also evolved in either constant or fluctuating temperature. We implemented competition experiments in two environments which matched the conditions during bacterial evolution, one with constant $\left(30^{\circ} \mathrm{C}\right)$ and the other with fluctuating $\left(20-30-40{ }^{\circ} \mathrm{C}\right.$, at two-hour intervals) temperature. Altogether we had eight different treatment combinations.

In the competition experiment, we used one clone per replicate population $(n=8)$ for each bacterial species. After the evolution (see "Study species"), clones were isolated from each of the populations and frozen at $-80{ }^{\circ} \mathrm{C}(1: 1$ in $80 \%$ glycerol). At first, we propagated all study species separately from frozen samples for three days at $30{ }^{\circ} \mathrm{C}$ and measured clones' inoculum sizes as optical densities (OD) in temperature-controlled spectrophotometers (Bioscreen $\mathrm{C}^{\circledR}$, Oy Growth Curves Ab, Ltd., Helsinki, Finland). After that, one clone from each $S$. marcescens population was chosen randomly to compete with one clone from each population of its competitor species in biculture. The initial invader-to-competitor ratio was 1:24. This experiment was repeated with all three competitor species (P. putida, P. fluorescens, N. capsulatum). In total there were 192 competition experiments.

The experimental microcosms were $15 \mathrm{ml}$ centrifuge tubes (Sarstedt, Numbrecht, Germany) containing $3 \mathrm{ml}$ of sterile nutrient broth medium (10 $\mathrm{g}$ of nutrient broth powder [Difco, Becton, Dickinson \& Company, Sparks, MD] and $1.25 \mathrm{~g}$ of yeast extract (Difco) in 11 of sterile $\mathrm{ddH}_{2} \mathrm{O}$ ). We initiated the competition experiments by pipetting $2 \mu \mathrm{l}$ of $S$. marcescens and $48 \mu 1$ of one of its competitor species into all of the 192 tubes. Species were inoculated concurrently so that there was an equal opportunity for consuming the resources. Half of the tubes were kept in constant $\left(30^{\circ} \mathrm{C}\right)$ and half in fluctuating 
(see "Study species") temperatures. The tube caps were kept loose to ensure the gas exchange. We allowed the species to compete for three days (72 h), after which we sampled $500 \mu \mathrm{l}$ of bacterial suspension from each tube into cryotubes containing $500 \mu \mathrm{l}$ of $80 \%$ glycerol and stored them at $-80{ }^{\circ} \mathrm{C}$ for later analysis.

\section{Determination of competitive success}

To determine the competitive success of $S$. marcescens, we counted the invader colonies in each sample after three days of competition. We plated all the 192 frozen samples in a random order. We used a standard dilution-series technique, i.e., we pipetted $100 \mu \mathrm{l}$ of thawed bacterial suspension into $900 \mu \mathrm{l}$ of sterile $\mathrm{ddH}_{2} \mathrm{O}$ and repeated the tenfold dilution six times to achieve $10^{-5}$-fold and $10^{-6}$-fold dilutions of the original samples. These dilutions allowed us to count separate colonies on agar plates. The discrimination of species, $S$. marcescens or other, was conducted on DNase test agar plates (Becton, Dickinson and Company, Sparks, MD; premade at Tammertutkan maljat, Tampere, Finland). DNase plates allow for separation of the invader colonies from the competitor species colonies because only $S$. marcescens can break down DNA enzymatically, which is seen as a clear halo around the colonies (Smith et al. 1969, Ketola et al. 2017). After two to three days of propagation at room temperature, we counted the $S$. marcescens colonies and all bacterial colonies on each plate to estimate the competitive success $(\mu)$ of the invader expressed as the proportion of $S$. marcescens colonies in the total colony count.

\section{Data analysis}

We tested the effect of the environment temperature during competition, as well as that of the evolution temperature of the invader and competitor species, on the competitive success of $S$. marcescens. We modeled the odds of encountering $S$. marcescens colonies in all bacterial colonies on a DNase agar plate. As we had a non-normal proportion data and the analysis included random effects, we analyzed the results with generalized linear mixed model (GLMMs; Bolker et al. 2009). We used a binomial error distribution and a logit link, and set the total number of colonies on a plate as a denominator to control for the total number of events in a trial. All analyses were run in SPSS ver. 24.0 (IBMSPSS, Chicago, IL).

The three fixed factors were the environment during competition, the evolution of the invader and the evolution of the competitor species, which all had two levels, constant and fluctuating temperatures. We fitted these three fixed factors, all their two-way interactions and the threeway interaction as explanatory variables. The identity of the $S$. marcescens clone, regardless of its evolution regime, and the identity of the competitor species were fitted as random factors. This was done to control for the non-independency of the observations, arising from the fact that the competitive success of the same invader clones was measured in two environments and against several competitor species. In addition, we also performed backward model selection for the full factorial model to find the most reduced model by removing effects for which $p>0.1$. This procedure did not change the biological interpretation of the results.

To test the sensitivity of the main results, we re-ran our model including also the fixed effect of competitor species' identity, and all possible two- to four-way interactions with other fitted factors. This allows test the responses of competitor species to the environment temperature during competition and the evolution temperature of the invader and its competitor species. In addition, we tested a model in which the inoculum sizes of both the competitor species' clones and the invaders' clones were added as covariates to control for the differences in starting cell densities. Moreover, using the data from Saarinen et al. (2018), we tested whether growth characteristics of the invader clones at nearly optimal thermal conditions $\left(30{ }^{\circ} \mathrm{C}\right)$, extreme thermal conditions $\left(20^{\circ} \mathrm{C}\right.$ and $\left.40{ }^{\circ} \mathrm{C}\right)$ or during thermal fluctuations (see "Study species") affected the competitive success of the invader by including them in the analysis as fixed covariates. As all additional analyses confirmed our findings from the full factorial model, in the following we present the results from the full factorial and 

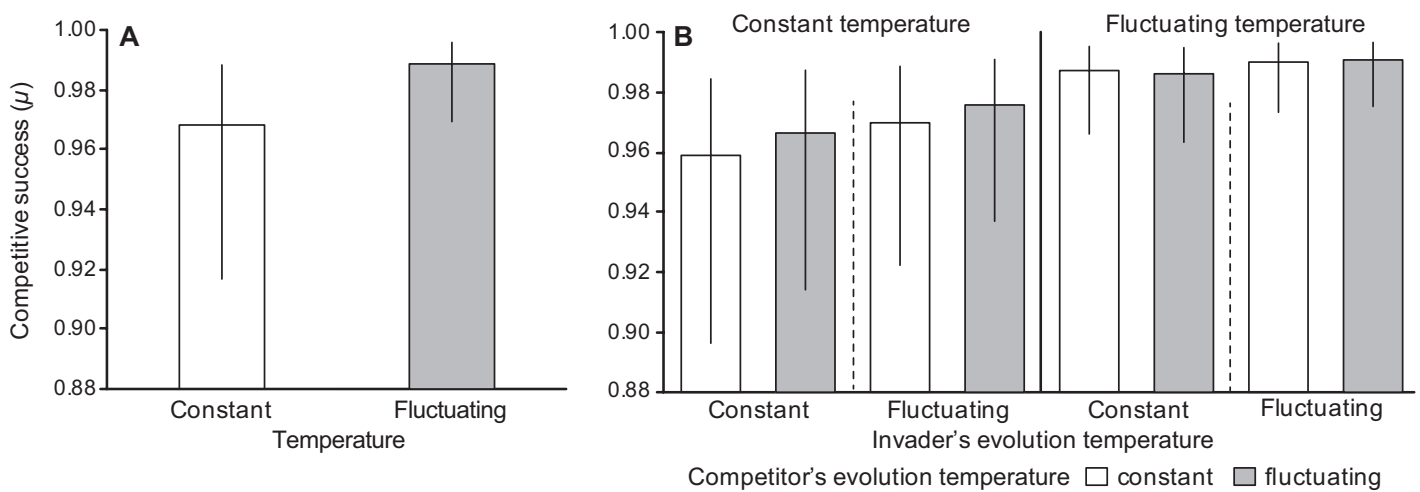

Fig. 1. The effects of $(\mathbf{A})$ the environment temperature during competition, and $(\mathbf{B})$ the three-way interaction of the environment temperature during competition, the invader's evolution temperature and the competitor's evolution temperature. Competitive success $(\mu)$ is the proportion of Serratia marcescens colonies in the total colony count after three days (72 h) of competition. Shown are the estimated marginal means with $95 \%$ confidence limits (CL).

the reduced models only. All count data, clones' inoculum sizes and additional data of maximum growth rates and yields are available at https:// doi.org/10.17011/jyx/dataset/68316.

\section{Results}

The full factorial model indicated high competitive success of $S$. marcescens after three days of competition (all-data mean $\mu=0.981$, $95 \% \mathrm{CL}=0.949-0.993)$. In all the studied species' pairs, there was clearly one factor, the environment temperature during competition, which had an effect on the competitive success. Serratia marcescens was more successful when the environment temperature during competition was fluctuating $(\mu=0.989,95 \% \mathrm{CL}=0.969$ $0.996)$ rather than constant $(\mu=0.968$, $95 \% \mathrm{CL}=0.917-0.988, p=0.001$ ) (Fig. 1A and Table 1). The invader's evolution temperature, the competitor's evolution temperature, as well as all the studied interactions had no effect on the competitive success of $S$. marcescens (Fig. 1B and Table 1). The random effect of the invader clones' identity was significant $\left(\sigma_{\text {logit }}^{2}=0.503\right.$, $\mathrm{SE}=0.205, Z=2.447, p=0.014)$, but the effect of the competitor species identity was non-significant $\left(\sigma_{\text {logit }}^{2}=0.271, \mathrm{SE}=0.275, Z=0.985\right.$, $p=0.325)$

The reduced model produced the same results. The only difference was that competitive

Table 1. The results of the generalized linear mixed model testing the effects of the environment temperature during competition, as well as the evolution temperature of the invader and the competitor species on the competitive success $(\mu)$ of Serratia marcescens after three days $(72 \mathrm{~h})$ of competition. Full model and the most reduced model after backward model selection with removal criterion of $p>0.1$; df1 was 1 for all factors and factor interactions.

\begin{tabular}{|c|c|c|c|c|c|c|}
\hline & \multicolumn{3}{|c|}{ Full model } & \multicolumn{3}{|c|}{ Reduced model } \\
\hline & $F$ & df2 & $p$ & $F$ & df2 & $p$ \\
\hline Environment during competition & 141.679 & 182 & 0.001 & 155.031 & 186 & 0.001 \\
\hline Invader's evolution & 0.792 & 14 & 0.389 & 0.815 & 14 & 0.382 \\
\hline Competitor's evolution & 1.533 & 182 & 0.217 & 3.184 & 186 & 0.076 \\
\hline Environment during competition $\times$ invader's evolution & 0.001 & 182 & 0.981 & & & \\
\hline Environment during competition $\times$ competitor's evolution & 1.624 & 182 & 0.204 & & & \\
\hline Invader's evolution $\times$ competitor's evolution & 0.256 & 182 & 0.614 & & & \\
\hline \multirow{2}{*}{ Environment during competition $\times$ invader's evolution $\times$ competitor's evolution } & 0.165 & 182 & 0.685 & & & \\
\hline & \multicolumn{3}{|c|}{$\mathrm{AIC}=1153.181$} & \multicolumn{3}{|c|}{$\mathrm{AIC}=1139.420$} \\
\hline
\end{tabular}


success of the invader seemed insignificantly higher when the competitor species had evolved in fluctuating $(\mu=0.982,95 \% \mathrm{CL}=0.952-$ 0.994) rather than in constant temperature $(\mu=0.980,95 \% \mathrm{CL}=0.945-0.993, p=0.076$; see Table 1). The AIC value for the reduced model was slightly better than that for the full model (see Table 1).

\section{Discussion}

Ongoing climate change is increasing fluctuations in global temperature (IPCC 2018), and therefore can potentially increase chances of successful species invasions now and in future (Stachowicz et al. 2002, Parepa et al. 2013). Despite that, very few studies have tested this prediction (Kreyling et al. 2008, Lee \& Gelembiuk 2008, Ketola et al. 2013, Saarinen et al. 2019). Experimental evaluation of the relative importance of ecological and evolutionary processes, which affect species' invasion success at different timescales is also lacking (Lee 2002, Facon et al. 2006). In our manipulative laboratory experiment, we quantified the effect of temperatures fluctuating on the ecological timescale and during the evolution of the invader and its competitor on the competitive success of the invader. We found that fast temperature fluctuations in the environment during competition clearly facilitated competitive success (Fig. 1A and Table 1). However, we did not detect any evidence for the effect of fluctuating temperatures during invaders' or its competitor's evolution, or the interactions between the studied factors on the outcome of the competition (Fig. 1B and Table 1).

In accordance with our hypothesis, $S$. marcescens was more successful, when the temperature during competition was fluctuating, rather than when it was constant (Fig. 1A and Table 1). This result indicates that disturbed environments are more prone to invasions than less disturbed, and is consistent with the results of previous studies carried out on plants and bacteria (Burke \& Grime 1996, Davis et al. 2000, Li \& Stevens 2012, Saarinen et al. 2019). Our study is among the first ones to show experimental evidence that fluctuations in temperature, not just in resource availability, could also affect species' competi- tive success, and potentially its ability to invade (Davis et al. 2000, Melbourne et al. 2007, Liu et al. 2012, Saarinen et al. 2019). Experiments providing data on fluctuations in other environmental factors and their interactions could offer interesting insights (Kreyling et al. 2008, Parepa et al. 2013). However, it should be remembered that disturbed and fluctuating environments may differ, and besides the anthropogenic pressure, there is also natural variation in environmental conditions which could affect species' invasion success (Ricciardi \& MacIsaac 2000, Winkler et al. 2008). Contrary to competitive exclusion, environmental fluctuations can also act as a factor promoting species' coexistence and maintaining diversity (Chesson 2000).

Unexpectedly, we found that evolution of $S$. marcescens in the fluctuating temperature had no effect on its competitive success (Fig. 1B and Table 1). This contradicts the notion that adaptation to fluctuating environments improves species' invasiveness (Lee \& Gelembiuk 2008). Some earlier studies found evidence for the higher invasion success of species and populations that evolved in disturbed habitats (Baker 1974, Winkler et al. 2008, Foucaud et al. 2010, Ketola et al. 2013, Saarinen et al. 2019). Also, permissively fluctuating temperatures can select for enhanced performance traits such as faster growth rate (Colinet et al. 2015). In our study, all bacterial cultures were initiated from clones of replicate populations which had evolved in specific environments. Even when starting from a homogeneous gene pool, the evolutionary time in an experimental setup closely similar to ours, is sufficient for adaptations to occur in response to selection (Ketola et al. 2013). This suggests that within a given time the selection for improved competitive ability was not very strong. Alternatively the lack of results could be due to clone-specific evolution of growth traits that could mask treatment effects. However, our additional analyses indicated that the competitive success of $S$. marcescens was not explained by its growth characteristics nor the ability to compete with other species (resource or interference competition).

Like invaders, competitor species could also benefit from the evolution in fluctuating environments (Saarinen et al. 2019). Contrary to our 
hypothesis, the results did not show higher competitive success of $S$. marcescens when the competitor species had evolved in a constant-temperature environment (Fig. 1B and Table 1). In the reduced model, the effect of the competitor's evolution temperature was insignificant ( $p=$ 0.076 ; see Table 1) but pointing to better competitive success of the invader when its competitor had evolved in a fluctuating-temperature environment. Whether the species' evolution in a constant thermal environment could affect their adaptation to thermally fluctuating environments is unclear. For example, in the long-term study of tobacco hornworm (Manduca sexta) (Kingsolver et al. (2009) did not find divergence in the responses of fluctuation- and constant-environment adapted populations to fast temperature fluctuations. In another experiment, populations of the pitcher plant mosquito (Wyeomyia smithii) from different geographic locations showed no genetic differences in their response to diurnal fluctuations (Ragland \& Kingsolver 2008). In both studies, the between-population differences in life-history traits were dependent on the mean temperature rather than the variation in temperature (Ragland \& Kingsolver 2008, Kingsolver et al. 2009). On the other hand, bacterial communities that had adapted to constant conditions were found to be more vulnerable to invasion, especially during the early stages of the experiment (Saarinen et al. 2019). More studies are needed to assess whether climate change can facilitate biological invasions by favoring invasive species over their native competitors (Stachowicz et al. 2002).

In addition, we found no effect of the interaction between the environment temperature during competition and that during the invader's evolution (Fig. 1B and Table 1). This contradicts the anthropogenically induced adaptation to invade hypothesis and the previous findings on invasive species, which show evidence that pre-adaptation of organisms to matching environmental conditions makes them more successful in invading new areas (Ricciardi \& MacIsaac 2000, Bossdorf et al. 2008, Winkler et al. 2008, Foucaud et al. 2010, Hufbauer et al. 2012, Hamilton et al. 2015). Along with this, none of the interactions were found to affect the competitive success of $S$. marcescens, pointing that the studied factors were not dependent on each other (see Fig. 1B and Table 1). Some studies found stronger evidence for these interactions, when the environmental conditions during invasion, the traits of the invader and the attributes of its native competitors acted together (Kreyling et al. 2008, Litchman 2010, Mächler \& Altermatt 2012, Saarinen et al. 2019).

In our study, the detection of the effects of temperature fluctuations on competition might have been confounded by the high overall competitive success of $S$. marcescens. Although we cannot separate the effect of the overall competitive success, it was clear that the temperature fluctuations during competition improved the success of the rare species. Previous studies carried out with the same bacterial strains, but setting $S$. marcescens against multiple species in the same culture and adding the competitor species frequently, did not find as pronounced invasions (Ketola et al. 2017, Saarinen et al. 2019). In addition, it could be argued that our artificial community was too simple as we used only one competitor species as "the community". Indeed, this is a simplification of nature, where also biodiversity and composition of native communities are assumed to affect their resistance to invasions (Davis 2009, Ketola et al. 2017). Recent studies highlighted, for example, the importance of multiple invaders and the abundance of the invader relative to that of the resident community as the primary drivers of invasion (Kinnunen et al. 2018, Rivett et al. 2018). However, the aim of our study was not to mimic the complexity of natural communities, but to efficiently test the theories of invasion biology (Naughton et al. 2015). The information emerging from microbial studies is also important since climate change is going to increase the spread of pathogens into new habitats and hosts (Bennett \& Hughes 2009, Litchman 2010, Ricciardi et al. 2017).

To conclude, we found that rapid temperature fluctuations during competition improve the competitive success of $S$. marcescens, pointing that the ecological context could be extremely important also in invasions (Shea \& Chesson 2002). Furthermore, we found no evidence that evolution under fluctuating conditions or competitors' lack of adaptation to tolerate temperature fluctuations makes the invader more successful. Our findings show that the current 
environmental variation resulting from climate change (IPCC 2018) could be the most prominent factor in promoting species' competitive success in fluctuating environments. However, further studies aiming at distinguishing the traits of the invader, the attributes of its native competitors and the environmental conditions during invasion, should be undertaken (Facon et al. 2006, Lee \& Gelembiuk 2008). Considering these factors together would allow us to make more accurate predictions of the species' ability to invade under novel, fluctuating climatic conditions (Kreyling et al. 2008, Litchman 2010, Mächler \& Altermatt 2012, Saarinen et al. 2019).

\section{Acknowledgements}

We thank Kati Saarinen for her work with the bacterial strains and the Centre of Excellence in Biological Interactions for funding and facilities. This work was financed by Societas Biologica Fennica Vanamo (E. Räsänen), and Academy of Finland Projects 278751 (T. Ketola) and 250248 (L. Lindstöm).

\section{References}

Baker, H. G. 1974: The evolution of weeds. - Annu. Rev. Ecol. Syst. 5: 1-24.

Bennett, A. F. \& Hughes, B. S. 2009: Microbial experimental evolution. - Am. J. Physiol. Regul. Integr. Comp. Physiol. 297: R17-R25, https://doi.org/10.1152/ ajpregu.90562.2008.

Bock, W. J. 1959: Preadaptation and multiple evolutionary pathways. - Evolution 13: 194-211.

Bolker, B. M., Brooks, M. E., Clark, C. J., Geange, S. W., Poulsen, J. R., Stevens, M. H. H. \& White, J. S. 2009: Generalized linear mixed models: a practical guide for ecology and evolution. - Trends Ecol. Evol. 24: 127-135.

Bossdorf, O., Lipowsky, A. \& Prati, D. 2008: Selection of preadapted populations allowed Senecio inaequidens to invade Central Europe. - Divers. Distrib. 14: 676-685.

Botero, C. A., Weissing, F. J., Wright, J. \& Rubenstein, D. R. 2015: Evolutionary tipping points in the capacity to adapt to environmental change. - Proc. Natl. Acad. Sci. USA 112: 184-189.

Burke, M. J. \& Grime, J. 1996: An experimental study of plant community invasibility. - Ecology 77: 776-790.

Chesson, P. 2000: Mechanisms of maintenance of species diversity. - Annu. Rev. Ecol. Syst. 31: 343-366.

Clements, D. \& Ditommaso, A. 2011: Climate change and weed adaptation: can evolution of invasive plants lead to greater range expansion than forecasted? - Weed Res. 51: $227-240$.
Colinet, H., Sinclair, B. J., Vernon, P. \& Renault, D. 2015: Insects in fluctuating thermal environments. - Аnnu. Rev. Entomol. 60: 123-140.

Davis, M. A. 2009: Invasion biology. — Oxford University Press, Oxford, UK.

Davis, M. A., Grime, J. P. \& Thompson, K. 2000: Fluctuating resources in plant communities: a general theory of invasibility. - J. Ecol. 88: 528-534.

Dukes, J. S. \& Mooney, H. A. 1999: Does global change increase the success of biological invaders? - Trends Ecol. Evol. 14: 135-139.

Duncan, A. B., Fellous, S., Quillery, E. \& Kaltz, O. 2011: Adaptation of Paramecium caudatum to variable conditions of temperature stress. - Res. Microbiol. 162: 939-944.

Facon, B., Genton, B. J., Shykoff, J., Jarne, P., Estoup, A. \& David, P. 2006: A general eco-evolutionary framework for understanding bioinvasions. - Trends Ecol. Evol. 21: $130-135$.

Foucaud, J., Orivel, J., Loiseau, A., Delabie, J.H., Jourdan, H., Konghouleux, D., Vonshak, M., Tindo, M., Mercier, J. \& Fresneau, D. 2010: Worldwide invasion by the little fire ant: routes of introduction and eco-evolutionary pathways. - Evol. Appl. 3: 363-374.

Hamilton, J. A., Okada, M., Korves, T. \& Schmitt, J. 2015: The role of climate adaptation in colonization success in Arabidopsis thaliana. - Mol. Ecol. 24: 2253-2263.

Hufbauer, R. A., Facon, B., Ravigné, V., Turgeon, J., Foucaud, J., Lee, C. E., Rey, O. \& Estoup, A. 2012: Anthropogenically induced adaptation to invade (AIAI): contemporary adaptation to human-altered habitats within the native range can promote invasions. - Evol. Appl. 5: 89-101.

IPCC 2018: Summary for policymakers. - An IPCC Special Report on the impacts of global warming of $1.5^{\circ} \mathrm{C}$ above pre-industrial levels and related global greenhouse gas emission pathways, in the context of strengthening the global response to the threat of climate change, sustainable development, and efforts to eradicate poverty, World Meteorological Organization, Geneva, Switzerland.

Kassen, R. 2002: The experimental evolution of specialists, generalists, and the maintenance of diversity. - J. Evolution Biol. 15: 173-190.

Ketola, T., Saarinen, K. \& Lindström, L. 2017: Propagule pressure increase and phylogenetic diversity decrease community's susceptibility to invasion. - BMC Ecol. 17: 15, https://doi.org/10.1186/s12898-017-0126-z.

Ketola, T., Mikonranta, L., Zhang, J., Saarinen, K., Örmälä, A., Friman, V., Mappes, J. \& Laakso, J. 2013: Fluctuating temperature leads to evolution of thermal generalism and preadaptation to novel environments. - Evolution 67: 2936-2944.

Kingsolver, J. G., Ragland, G. J. \& Diamond, S. E. 2009: Evolution in a constant environment: thermal fluctuations and thermal sensitivity of laboratory and field populations of Manduca sexta. - Evolution 63: 537-541.

Kinnunen, M., Dechesne, A., Albrechtsen, H. J. \& Smets, B. F. 2018: Stochastic processes govern invasion success in microbial communities when the invader is phylogenetically close to resident bacteria. - ISME J. 12: 2748-2756. 
Kreyling, J., Beierkuhnlein, C., Ellis, L. \& Jentsch, A. 2008: Invasibility of grassland and heath communities exposed to extreme weather events - additive effects of diversity resistance and fluctuating physical environment. Oikos 117: 1542-1554.

Kristensen, T. N., Ketola, T. \& Kronholm, I. 2018: Adaptation to environmental stress at different timescales. - Ann. New York Acad. Sci., https://doi.org/10.1111/nyas.13974.

Lee, C. E. 2002: Evolutionary genetics of invasive species. - Trends. Ecol. Evol. 17: 386-391.

Lee, C. E. \& Gelembiuk, G. W. 2008: Evolutionary origins of invasive populations. - Evol. Appl. 1: 427-448

Levins, R. 1968: Evolution in changing environments: some theoretical explorations. - Princeton University Press, Princeton, NJ.

Li, W. \& Stevens, M. H. H. 2012: Fluctuating resource availability increases invasibility in microbial microcosms. - Oikos 121: 435-441.

Litchman, E. 2010: Invisible invaders: non-pathogenic invasive microbes in aquatic and terrestrial ecosystems. Ecol. Lett. 13: 1560-1572.

Liu, M., Bjørnlund, L., Rønn, R., Christensen, S. \& Ekelund, F. 2012: Disturbance promotes non-indigenous bacterial invasion in soil microcosms: analysis of the roles of resource availability and community structure. $-P L o S$ ONE 7(10): e45306, https://doi.org/10.1371/journal. pone. 0045306 .

Marvier, M., Kareiva, P. \& Neubert, M. G. 2004: Habitat destruction, fragmentation, and disturbance promote invasion by habitat generalists in a multispecies metapopulation. - Risk Anal. 24: 869-878.

Melbourne, B. A., Cornell, H. V., Davies, K. F., Dugaw, C. J., Elmendorf, S., Freestone, A. L., Hall, R. J., Harrison, S., Hastings, A. \& Holland, M. 2007: Invasion in a heterogeneous world: resistance, coexistence or hostile takeover? - Ecol. Lett. 10: 77-94.

Meyers, L. A., Ancel, F. D. \& Lachmann, M. 2005: Evolution of genetic potential. - PLoS Comput. Biol. 1(3): e32, https://doi.org/10.1371/journal.pcbi.0010032.

Mooney, H. A. \& Cleland, E. E. 2001: The evolutionary impact of invasive species. - Proc. Natl. Acad. Sci. USA 98: 5446-5451.

Mächler, E. \& Altermatt, F. 2012: Interaction of species traits and environmental disturbance predicts invasion success of aquatic microorganisms. - PLoS ONE 7(9): e45400, https://doi.org/10.1371/journal.pone.0045400.

Naughton, H., Alexandrou, M., Oakley, T. \& Cardinale, B. 2015: Phylogenetic distance does not predict competition in green algal communities. - Ecosphere 6(7): 116, http://dx.doi.org/10.1890/ES14-00502.1.
Parepa, M., Fischer, M. \& Bossdorf, O. 2013: Environmental variability promotes plant invasion. - Nat. Commun. 4, 1604, https://doi.org/10.1038/ncomms2632.

Ragland, G. J. \& Kingsolver, J. G. 2008: The effect of fluctuating temperatures on ectotherm life-history traits: comparisons among geographic populations of Wyeomyia smithii. — Evol. Ecol. Res. 10: 29-44.

Ricciardi, A. \& MacIsaac, H. J. 2000: Recent mass invasion of the North American Great Lakes by Ponto-Caspian species. - Trends Ecol. Evol. 15: 62-65.

Ricciardi, A., Blackburn, T. M., Carlton, J. T., Dick, J. T., Hulme, P. E., Iacarella, J. C., Jeschke, J. M., Liebhold, A. M., Lockwood, J. L., MacIsaac, H. J., Pyšek, P., Richardson, D. M., Ruiz, G. M., Simberloff, D., J. Sutherland, W. J., Wardle, D. A. \& Aldridge, D. C. 2017 : Invasion science: a horizon scan of emerging challenges and opportunities. - Trends Ecol. Evol. 32: 464-474.

Rivett, D. W., Jones, M. L., Ramoneda, J., Mombrikotb, S. B., Ransome, E. \& Bell, T. 2018: Elevated success of multispecies bacterial invasions impacts community composition during ecological succession. - Ecol. Lett. 21: $516-524$.

Saarinen, K., Lindström, L. \& Ketola, T. 2019: Invasion triple trouble: Environmental fluctuations, fluctuation-adapted invaders and fluctuation-mal-adapted communities all govern invasion success. - BMC Evol. Biol. 19: 42, https://doi.org/10.1186/s12862-019-1348-9.

Saarinen, K., Laakso, J., Lindström, L. \& Ketola, T. 2018: Adaptation to fluctuations in temperature by nine species of bacteria. - Ecol. Evol. 8: 2901-2910.

Shea, K. \& Chesson, P. 2002: Community ecology theory as a framework for biological invasions. - Trends Ecol. Evol. 17: 170-176.

Smith, P. B., Hancock, G. A. \& Rhoden, D. L. 1969: Improved medium for detecting deoxyribonuclease-producing bacteria. - Appl. Microbiol. 18: 991-993.

Stachowicz, J. J., Terwin, J. R., Whitlatch, R. B. \& Osman, R. W. 2002: Linking climate change and biological invasions: ocean warming facilitates nonindigenous species invasions. - Proc. Natl. Acad. Sci. USA 99: 15497-15500.

Winkler, G., Dodson, J. J. \& Lee, C. E. 2008: Heterogeneity within the native range: population genetic analyses of sympatric invasive and noninvasive clades of the freshwater invading copepod Eurytemora affinis. - Mol. Ecol. 17: 415-430.

Zerebecki, R. A. \& Sorte, C. J. 2011: Temperature tolerance and stress proteins as mechanisms of invasive species success. - PLoS ONE 6(4): e14806, https://doi. org/10.1371/journal.pone.0014806. 